\title{
Tips for training non-native English speakers
}

\author{
LaTesa Hughes, RLAT, DVM, MS
}

Communication is the process by which we give and get information. Successful communication occurs when there is understanding. My favorite quote from Rob Allen, trainer and author, is "a trainer's primary goal is simple: we want participants to remember the material we present. Otherwise, why teach it?" So true! If this is not our goal, our training session is a waste of time for both parties. I am a veterinarian at the US National Institutes of Health, where we have a diversity of languages within our animal care and researcher population. Over the years, through my animal care experiences and Teachers of English to Speakers of Other Languages (TESOL) training, I have added a few tools to my toolbox that help make my interactions with non-native English speakers (NNESs) more fruitful. Here I share some of them with my fellow lab animal community members.

Don't address the pause. During oral instruction, give the trainees time to process what you have said. Some people become uncomfortable when there is silence and try to fill the space with 'noise'. But trainees who are NNESs may be taking the words you have said and translating them into their native language... and then translating their response back again. This process takes time; their fluency with the English language may dictate how long.

Stay on your toes! Be aware of the use of idioms, idiomatic expressions, slang and phrasal verbs. Translation of these figures of speech may be difficult for NNESs, and standard dictionaries may not be helpful.

Check for understanding. Do not assume that because the trainees are nodding their

Hughes is veterinarian at the US National Institutes of Health, Bethesda, MD. heads without asking any questions, all the information has been fully understood. Be specific and do not hesitate to provide information more than once. It may be more helpful to rephrase rather than repeat. Written follow-ups, if feasible, can also be useful.

Appreciate where they learned English. If NNESs learned English in their home countries, their teachers may have passed along their native accents. The American accent may be different from anything they have ever heard.

Be patient. As managers and trainers, we learn early on that patience is required when training everyone. Be their personal cheerleader!

Keep readers in mind when writing training materials. Several short sentences are easier to understand than one long sentence with a bunch of commas and semi-colons. Aim to use fewer than 20 words per sentence. Check the readability statistics of training materials to evaluate the reading comprehension difficulty. The Flesch-Kincaid Grade level shows the reading grade level of the document. Aim for the eighth-grade (8.0) level when writing for the public and perhaps lower for NNESs. The Flesch Reading Ease score indicates the comprehension level of the document; the higher the score, the easier to read. Aim for a score of 60 or higher. This column is written at a eighth-grade level with a readability score of $\sim 60$. Note that these scores do not account for the technical vocabulary we use in the lab animal and research community.

Use automated grammar and style checkers. Take advantage of the automated grammar- and style-check functions included with many computer software packages. These features will check documents for common errors in punctuation and sentence structure, such as fragments, run-ons, jargon,

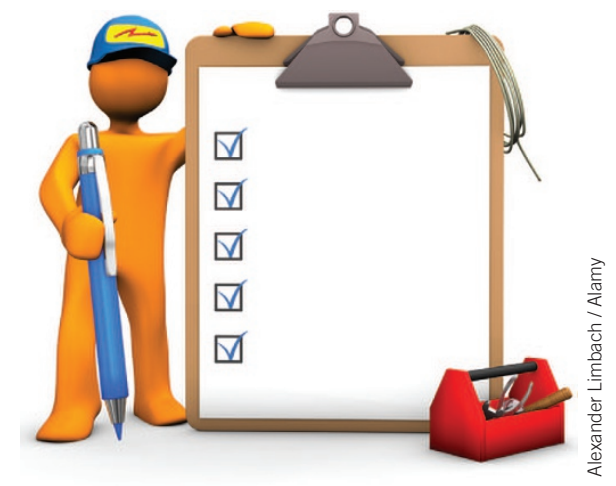

length, wordiness and unclear phrasing. Excellent tools!

Try learning a new language yourself. Learn a little bit of the native languages of the NNESs you are training; this does so much for both parties. It gives them an opportunity to practice because they have to teach you in English. It shows you are interested in them and their challenges. It puts you in their shoes (oh, an idiom; it is so hard not to use them) and allows you to empathize with them. It gives you a chance to learn something new-as trainers, we love to learn new things, right? Currently, I can have a short conversation in ten different languages. How awesome is that?! Lastly, it shows that you can be vulnerable and yet comfortable with that vulnerability. This may help NNESs feel more comfortable and grow more receptive to training.

Consider TESOL training or certification. Find out if your local community college has a TESOL program. Look for adult education associations or councils in your area. They may offer free workshops where you can pick up some great tips from the experts!

Do you have other tools? If so, I encourage you to share them. The Laboratory Animal Welfare and Training Exchange (http://www. lawte.org/) provides an excellent venue for fellow trainers, as well as managers and supervisors, to exchange ideas. 